\title{
Dissecting pseudoaneurysms: predictors of symptom occurrence, enlargement, clinical outcome, and treatment
}

\author{
Badih Daou, MD, Christine Hammer, MD, Nohra Chalouhi, MD, Robert M. Starke, MD, \\ Pascal Jabbour, MD, Robert H. Rosenwasser, MD, and Stavropoula Tjoumakaris, MD
}

Department of Neurosurgery, Thomas Jefferson University and Jefferson Hospital for Neuroscience, Philadelphia, Pennsylvania

OBJECTIVE Dissection of the carotid and vertebral arteries can result in the development of aneurysmal dilations. These dissecting pseudoaneurysms can enlarge and cause symptoms. The objective of this study is to provide insight into the progression of dissecting pseudoaneurysms and the treatments required to manage them.

METHODS A review of the electronic medical records was conducted to detect patients with carotid and vertebral artery dissection. An imaging review was conducted to identify patients with dissecting pseudoaneurysms. One hundred twelve patients with 120 dissecting pseudoaneurysms were identified. Univariate and multivariate analyses were conducted to assess the factors associated with undergoing further interventions other than medical treatment, pseudoaneurysm enlargement, pseudoaneurysms resulting in ischemic and nonischemic symptoms, and clinical outcome.

RESULTS Overall, $18.3 \%$ of pseudoaneurysms were intracranial and $81.7 \%$ were extracranial, and the average size was $7.3 \mathrm{~mm}$. The mean follow-up time was 29.3 months; $3.3 \%$ of patients had a recurrent transient ischemic attack, no patients had a recurrent stroke, and $14.2 \%$ of patients had recurrence of nonischemic symptoms (headache, neck pain, Horner syndrome, or cranial nerve palsy). Follow-up imaging demonstrated that $13.8 \%$ of pseudoaneurysms had enlarged, $30.2 \%$ had healed, and $56 \%$ had remained stable. In total, $20.8 \%$ of patients had an intervention other than medical treatment. Interventions included stenting, coiling, flow diversion, and clipping. Predictors of intervention included increasing size, size $>10 \mathrm{~mm}$, location in the $\mathrm{C}_{2}$ (petrous) segment of the internal carotid artery (ICA), younger age, hyperlipidemia, pseudoaneurysm enlargement, and any symptom development. Significant predictors of enlargement included smoking, history of trauma, $\mathrm{C}_{2}$ location, hyperlipidemia, and larger initial pseudoaneurysm size. Predictors of pseudoaneurysm resulting in recurrent ischemic and nonischemic symptoms included increasing size and location in the petrous segment of the ICA. Smoking was a predictor of unfavorable outcome.

CONCLUSIONS Dissecting pseudoaneurysms have a benign course and most will not cause symptoms or enlarge on follow-up. Medical treatment can be a sufficient, initial treatment for dissecting pseudoaneurysms.

http://thejns.org/doi/abs/10.3171/2015.10.JNS151846

KEY WORDS carotid; dissection; pseudoaneurysm; vertebral; vascular disorders

$\mathrm{D}$ ISSECTION of the carotid and vertebral arteries is an important cause of stroke in young patients, accounting for up to $25 \%$ of stroke cases in this age group. ${ }^{24}$ Dissection can be intracranial or extracranial, with the latter being more common. It can occur spontaneously, be associated with certain medical conditions including connective tissue disorders (fibromuscular dysplasia, Ehlers-Danlos and Marfan syndromes), or occur after a traumatic event. , 24,29 $^{2}$ Dissection of the carotid and vertebral arteries can result in the development of aneurysmal dilations at the site of dissection, especially in the case of subadventitial dissections. ${ }^{4}$ These are mostly pseudoaneu- rysms and are known as dissecting pseudoaneurysms. The incidence of dissections resulting in pseudoaneurysm formation has been reported to range from $5 \%$ to $40 \% .{ }^{12}$ They can be saccular or fusiform and can have different presentations depending on the location. ${ }^{30}$ They can be observed as an acute event or they may be observed on follow-up with MR angiography (MRA), CT angiography, or digital subtraction angiography ${ }^{14,19,22}$ The problem with dissecting pseudoaneurysms is that they carry the potential to enlarge and become symptomatic. The treatments for dissections with coexisting pseudoaneurysms are less well established, with some authors suggesting a benign nature of

ABBREVIATIONS ICA = internal carotid artery; MRA = MR angiography; $\mathrm{mRS}=$ modified Rankin Scale; $\mathrm{TIA}=$ transient ischemic attack.

SUBMITTED August 7, 2015. ACCEPTED October 2, 2015.

INCLUDE WHEN CITING Published online January 29, 2016; DOI: 10.3171/2015.10.JNS151846. 
these aneurysmal structures and thus only requiring medical treatment with anticoagulation or antiplatelet agents, while other authors recommend surgical or endovascular interventions in order to eliminate the risk of aneurysm rupture or thrombus formation and embolization leading to transient ischemic attack (TIA) and stroke. ${ }^{1,21,25,30}$ The most widely used endovascular intervention is stenting, as its safety and efficacy have been increasingly supported by evidence in the literature. ${ }^{13,27,28}$ The objective of this study is to provide insight on the progression of traumatic and spontaneous dissecting pseudoaneurysms, the treatments required to manage them (medical vs surgical), and identify factors that can be associated with pseudoaneurysm enlargement, ischemic and nonischemic symptom development, and clinical outcome in patients harboring these structures.

\section{Methods \\ Patients}

The study protocol was approved by our university's institutional review board. A review of the electronic medical records was conducted. The search criteria included patients with the diagnosis of carotid and vertebral dissection (ICD-9 codes 443.21 and 443.24). Three hundred seventy patients with carotid and vertebral artery dissection were identified. A review of the images (including MR angiograms, CT angiograms, and angiograms) was conducted to identify patients with the diagnosis of dissecting pseudoaneurysms. The diagnosis of pseudoaneurysms and the changes in size were made by specialized neuroradiologists. One hundred twelve patients with 120 dissecting pseudoaneurysms were identified.

\section{Outcomes}

All patients with dissecting pseudoaneurysms were placed on an antiplatelet or anticoagulation agent to prevent thromboembolic complications. The main outcomes of interest in this study included frequency of pseudoaneurysm development, frequency and predictors of pseudoaneurysm enlargement, frequency and predictors of pseudoaneurysms resulting in ischemic and nonischemic symptoms, frequency and predictors of poor outcomes as evaluated at the latest follow-up using the modified Rankin Scale (mRS), and the proportion of patients who had recurrent ischemic or hemorrhagic events. Symptoms and clinical outcomes were determined by the treating neurosurgeon. Another goal of this study was to evaluate the frequency of patients who underwent interventions other than medical treatment using endovascular or surgical approaches. In general, interventions were performed on enlarging pseudoaneurysms and symptomatic pseudoaneurysms. Patients with stable and healing pseudoaneurysms were less likely to be considered candidates for surgical or endovascular interventions. All interventions were performed by 1 of 4 neurosurgeons trained in both endovascular and open vascular approaches. Since treatment preferences, thresholds, and practice patterns may vary, we evaluated the common factors that were more likely to be present in patients who underwent an intervention.

\section{Studied Variables}

The dependent variables that were assessed for significance included age, sex, smoking, hypertension, history of stroke, history of cardiovascular disease, history of hyperlipidemia, history of diabetes, history of trauma, aneurysm location, involved segment of the internal carotid artery (according to the Bouthillier classification) or vertebral artery, intracranial versus extracranial location, aneurysm shape (saccular or fusiform), and aneurysm size.

\section{Statistical Analysis}

Data are presented as the mean and range for continuous variables, and as the frequency for categorical variables. Analysis was carried out using the unpaired t-test, chi-square, and Fisher exact tests. The assessment of the means of more than 2 groups was carried out by analysis of variance with post hoc analysis and Bonferroni correction as appropriate. Univariate analysis was used to test covariates predictive of the following dependent variables: undergoing an intervention (other than a medical intervention), aneurysm enlargement, pseudoaneurysm resulting in ischemic symptoms alone, overall occurrence of ischemic and nonischemic symptoms, and unfavorable outcome (mRS Score 2-6). Interaction and confounding were assessed through stratification and relevant expansion covariates. Factors predictive in the univariate analysis $(\mathrm{p}<$ $0.15)$ were entered into a multivariate logistic regression analysis, and $\mathrm{p}$ values of $\leq 0.05$ were considered statistically significant. Statistical analysis was carried out with Stata version 10.0 .

\section{Results \\ Baseline Characteristics}

The mean patient age was 48.4 years, $39.2 \%$ of patients were men, and $60.8 \%$ were women. The mean follow-up time was 29.3 months, $28.6 \%$ were smokers, $44.2 \%$ had a history of hypertension, $13.3 \%$ had a history of stroke, $15 \%$ had a history of cardiovascular disease, $24.2 \%$ had a history of hyperlipidemia, and $6.7 \%$ had a history of diabetes (Table 1). Of the 370 patients identified with carotid and vertebral artery dissections, $30.3 \%$ developed 1 or more pseudoaneurysms. Pseudoaneurysms developed in $39 \%$ of patients with carotid artery dissection and in $23 \%$ of patients with vertebral artery dissection; $30.8 \%$ of patients had a history of head trauma prior to the occurrence of the pseudoaneurysm; $53.4 \%$ of pseudoaneurysms were located in the $\mathrm{C}_{1}$ division (cervical) of the internal carotid artery (ICA), $20.8 \%$ were located in the $\mathrm{C}_{2}$ (petrous) division of the ICA, $10.8 \%$ of patients had an aneurysm in the $\mathrm{V}_{2}$ segment of the vertebral artery, $6.7 \%$ in the $\mathrm{V}_{3}$ segment of the vertebral artery, $5.8 \%$ in the $\mathrm{V}_{4}$ segment of the vertebral artery, $0.83 \%$ of patients in the $\mathrm{V}_{1}$ segment, $0.83 \%$ of patients in the $\mathrm{C}_{6}$ division of the ICA (superior hypophyseal artery), and $0.83 \%$ of patients in the anterior cerebral artery (Table 2). Overall, $45 \%$ occurred on the right and $55 \%$ occurred on the left; $81.7 \%$ of pseudoaneurysms were extracranial and $18.3 \%$ were intracranial, $82.5 \%$ were saccular, and $17.5 \%$ were fusiform in shape. The average pseudoaneurysm size was $7.3 \mathrm{~mm}$ (range 2-25 mm). These structures were detected at the first radiological evaluation in 74 patients $(61.7 \%)$. As an initial 
TABLE 1. Patient characteristics

\begin{tabular}{lc}
\hline \multicolumn{1}{c}{ Characteristic } & Value \\
\hline Mean age & $48.4 \mathrm{yrs}$ \\
\hline Mean follow-up time & $29.3 \mathrm{mos}$ \\
\hline Male/female & $39.2 \% / 60.8 \%$ \\
\hline Smokers & $28.6 \%$ \\
\hline Hypertension & $44.2 \%$ \\
\hline Prior stroke & $13.3 \%$ \\
\hline Cardiovascular disease & $15 \%$ \\
\hline Hyperlipidemia & $24.2 \%$ \\
\hline Diabetes & $6.7 \%$ \\
\hline History of trauma & $30.8 \%$ \\
\hline Extracranial/intracranial & $81.7 \% / 18.3 \%$ \\
\hline Saccular/fusiform & $82.5 \% / 17.5 \%$ \\
\hline Mean aneurysm size & $7.3 \mathrm{~mm}$ \\
\hline
\end{tabular}

management, all patients were placed on a regimen of antithrombotic treatment. This consisted of antiplatelet treatment in $59 \%$ of patients (aspirin in $17 \%$, Plavix in $10.7 \%$, aspirin and Plavix in 31.25\%), heparin and warfarin in $26.8 \%$, and combined antiplatelet and anticoagulation agents in $14.3 \%$ of patients. The choice of antithrombotic treatment was decided by the treating physician. Patients in whom pseudoaneurysms were detected early following dissection were more likely to be on anticoagulation or combined treatment, whereas patients who had their pseudoaneurysm detected over a longer time period following dissection were more likely to be on antiplatelet treatment. Antithrombotic treatment strategies were distributed similarly among patients with or without history of trauma (among trauma patients, 55\% were on antiplatelet treatment and $45 \%$ were on anticoagulation or combined treatment; among patients without trauma, 60\% were on antiplatelet treatment and $40 \%$ were on anticoagulation or combined treatment), extracranial or intracranial pseudoaneurysms (among extracranial pseudoaneurysms, $56 \%$ were on antiplatelet treatment and $44 \%$ were on anticoagulation or combined treatment; among intracranial pseudoaneurysms, $68 \%$ were on antiplatelet treatment and $32 \%$ were on anticoagulation or combined treatment), and carotid or vertebral artery involvement (among those with carotid artery involvement, $56 \%$ were on antiplatelet treatment and $44 \%$ were on anticoagulation or combined treatment; among those with vertebral artery involvement, $64 \%$ were on antiplatelet treatment and $36 \%$ were on anticoagulation or combined treatment).

\section{Outcomes}

Only 4 patients had a recurrent TIA (3.3\%), with none having a recurrent stroke within the distribution of the dissected artery/pseudoaneurysm or hemorrhagic complications. In total, $14.2 \%$ of patients had recurrence of nonischemic symptoms during follow-up. Symptoms included recurrent and worsening headache, neck pain, Horner syndrome, and cranial nerve palsy. Overall, $13.8 \%$ of patients were found to have enlargement on follow-up imaging, 30.2\% healed, and 56\% remained stable. Four patients did not have follow-up imaging. In total, $20.8 \%$ of patients
TABLE 2. Pseudoaneurysm location

\begin{tabular}{cc}
\hline \multicolumn{1}{c}{ Location } & Percentage \\
\hline ICA & \\
\hline $\mathrm{C}_{1}$ segment (cervical) & 53.4 \\
\hline $\mathrm{C}_{2}$ segment (petrous) & 20.8 \\
\hline $\mathrm{C}_{6}$ segment (ophthalmic) & 0.83 \\
\hline Anterior cerebral artery & 0.83 \\
\hline Vertebral artery & \\
\hline $\mathrm{V}_{1}$ segment & 0.83 \\
\hline $\mathrm{V}_{2}$ segment & 10.8 \\
\hline $\mathrm{V}_{3}$ segment & 6.7 \\
\hline $\mathrm{V}_{4}$ segment & 5.8 \\
\hline
\end{tabular}

underwent an intervention other than medical treatment alone to manage pseudoaneurysms. Of these patients, 15 patients were treated with stenting $(60 \%), 4$ patients were managed with stent-assisted coiling (16\%), 3 patients were treated with coiling alone (12\%), 2 patients were treated with a Pipeline Embolization Device (8\%), and 1 patient was treated with clipping (4\%). In the 25 patients who had an intervention, only 1 patient had a $10 \%$ recurrence of the pseudoaneurysm (after stent-assisted coiling). All others had complete obliteration of the pseudoaneurysm. The mean time from diagnosis of the pseudoaneurysm to intervention, if any, was 3.5 months. The median mRS score at the latest clinical follow-up was 0 (Table 3).

\section{Factors Associated With Pseudoaneurysm Enlargement}

In univariate analysis, smoking $(\mathrm{OR} 3.2, \mathrm{p}=0.035,95 \%$ CI 1.08-9.4), hyperlipidemia (OR 2.9, $\mathrm{p}=0.053,95 \%$ CI

TABLE 3. Outcomes

\begin{tabular}{cc}
\hline Characteristic & Value \\
\hline Occurrence of symptoms, \% & 17.5 \\
\hline Extracranial pseudoaneurysms & 14.3 \\
\hline Intracranial pseudoaneurysms & 31.8 \\
\hline Traumatic pseudoaneurysms & 16.2 \\
\hline Spontaneous pseudoaneurysms & 18 \\
\hline Pseudoaneurysm enlargement, \% & 13.8 \\
\hline Extracranial pseudoaneurysms & 12.2 \\
\hline Intracranial pseudoaneurysms & 18.2 \\
\hline Traumatic pseudoaneurysms & 21.6 \\
\hline Spontaneous pseudoaneurysms & 9.6 \\
\hline Intervention other than medical treatment, \% & 20.8 \\
\hline Extracranial pseudoaneurysms & 17.3 \\
\hline Intracranial pseudoaneurysms & 36.4 \\
\hline Traumatic pseudoaneurysms & 27 \\
\hline Spontaneous pseudoaneurysms & 18.1 \\
\hline Median mRS score at last follow-up & 0 \\
\hline Extracranial pseudoaneurysms & 0 \\
\hline Intracranial pseudoaneurysms & 0 \\
\hline Traumatic pseudoaneurysms & 0 \\
\hline Spontaneous pseudoaneurysms & 0 \\
\hline
\end{tabular}


0.99-8.88), initial size (OR 1.3, $\mathrm{p}=0.001,95 \%$ CI 1.2$1.6)$, initial size $>10 \mathrm{~mm}(\mathrm{OR} 10.5, \mathrm{p}=0.000,95 \% \mathrm{CI}$ 3-36.4), and location in the $\mathrm{C}_{2}$ segment of the ICA (OR $5.7, \mathrm{p}=0.002,95 \%$ CI 1.86-17.6) were significant predictors of enlargement. In the multivariate analysis, smoking (OR 15.8, $\mathrm{p}=0.018,95 \%$ CI 1.6-156.6), hyperlipidemia (OR $17.5, \mathrm{p}=0.014,95 \%$ CI 1.8-171.9), history of trauma (OR 26.8, $\mathrm{p}=0.009,95 \% \mathrm{CI} 2.3-313.3)$, location in the $\mathrm{C}_{2}$ (petrous) segment of the ICA (OR 15, $\mathrm{p}=0.016,95 \%$ CI 1.7-141.7), and larger initial pseudoaneurysm size (OR $1.44, \mathrm{p}=0.001,95 \%$ CI 1.16-1.79) were significantly associated with pseudoaneurysm enlargement.

\section{Factors Associated With Endovascular or Surgical Intervention}

In the univariate analysis, hyperlipidemia (OR 2.7, $\mathrm{p}=0.042,95 \%$ CI 1.04-6.9), increasing size (OR 1.28, $\mathrm{p}=0.001,95 \%$ CI 1.1-1.5), size $>10 \mathrm{~mm}(\mathrm{OR} 7.4, \mathrm{p}=$ $0.000,95 \%$ CI 2.5-21.7), and occurrence of any ischemic and nonischemic symptoms (OR 32, p =0.000, 95\% CI 9.5-107.9) were significant predictors of intervention. In the multivariate analysis, increasing pseudoaneurysm size (OR 1.3, $\mathrm{p}=0.001,95 \%$ CI 1.12-1.54), $\mathrm{C}_{2}$ location (OR 5.4, $\mathrm{p}=0.012,95 \%$ CI 1.4-20.1), decreasing age (OR 1.07, $\mathrm{p}$ $=0.04,95 \%$ CI 1.002-1.14), and hyperlipidemia (OR 7.2, $\mathrm{p}=0.02,95 \%$ CI 1.4-38.3) were significant predictors of endovascular or surgical intervention. Aneurysms that resulted in ischemic or nonischemic symptoms (OR 58, p < $0.001,95 \%$ CI 8-424) and those that enlarged (OR 32, $\mathrm{p}<$ $0.001,95 \%$ CI 9.5-107.9) had higher rates of intervention as well.

\section{Factors Associated With Ischemic and Nonischemic Symptoms}

Analysis of the patients with ischemic symptoms alone (only 4 patients) did not reveal any significant factors associated with the occurrence of these events. In the univariate analysis, pseudoaneurysms located in the $\mathrm{C}_{2}$ segment (OR 3.9, $\mathrm{p}=0.009,95 \%$ CI 1.4-10.7) had higher odds of resulting in combined ischemic and nonischemic symptoms. In the multivariate analysis, larger pseudoaneurysms (OR 1.1, p $=0.037,95 \%$ CI 1.005-1.21) located in the $\mathrm{C}_{2}$ segment of the ICA (OR 4.4, $\mathrm{p}=0.026,95 \%$ CI 1.216.1) were significantly associated with the occurrence of overall ischemic and nonischemic symptoms related to the pseudoaneurysm.

\section{Factors Associated With Clinical Outcome}

In both the univariate and multivariate analyses, smoking was the only statistically significant factor associated with poor functional outcome (mRS Score 2-6) (OR 3.6, $\mathrm{p}=0.04,95 \%$ CI 1.02-13) (Table 4).

\section{Discussion}

In total, $30.3 \%$ of patients with carotid and vertebral dissections developed 1 or more pseudoaneurysms. Pseudoaneurysms were more likely to develop with carotid dissections (39\%) than vertebral dissections (23\%). Similarly, in the CADISP (Cervical Artery Dissection Ischemic
Stroke Patients) study, Debette et al. reported that pseudoaneurysms were more common with carotid dissections than with vertebral artery dissections. ${ }^{7}$ Guillon et al. recorded pseudoaneurysm development in $27.5 \%$ of dissection patients. ${ }^{12}$ In general, our results are consistent with previous studies that reported a rate of pseudoaneurysm development in patients with carotid and vertebral dissection ranging from $5 \%$ to $40 \% .^{12,30}$ The rate of pseudoaneurysm development was similar for trauma (35.2\%) and nontrauma patients $(31.3 \%)$ in this study. These structures were only detected at the first radiological evaluation in $61.7 \%$ of patients. A large number of pseudoaneurysms $(38.3 \%)$ were detected at the later stages of evaluation, which reinforces the need to repeat MRA or obtain an angiogram, if not already performed, to detect these structures.

In total, $13.8 \%$ of pseudoaneurysms were found to have enlarged on follow-up imaging, whereas $30.2 \%$ were found to have healed and $56 \%$ were stable on follow-up. Touzé et al. reported that of 40 dissecting pseudoaneurysms, none enlarged during follow-up. ${ }^{30}$ Similarly, Guillon et al. did not observe any increase in size in 20 pseudoaneurysms. ${ }^{12}$ Djouhri et al. followed 26 dissecting pseudoaneurysms for a mean of 41 months using MRA and found that none of the aneurysms enlarged with most remaining stable (20 of 26 aneurysms), 4 decreasing from their original size, and 2 had resolved. ${ }^{8}$ While the percentage of pseudoaneurysm enlargement in our study is low, it is not negligible in comparison with other studies. Follow-up imaging to observe enlargement would be warranted since some aneurysmal forms can enlarge, as evidenced by this study.

Patients with a history of tobacco smoking, hyperlipidemia, larger initial pseudoaneurysm size, pseudoaneurysms located in the $\mathrm{C}_{2}$ (petrous) segment of the ICA, and traumatic dissecting pseudoaneurysms had higher odds of aneurysm enlargement. Smoking has been shown to decrease the activity of alpha-1 antitrypsin, leading to a disruption of the integrity of connective tissue and resulting in increased aneurysm formation and growth. ${ }^{26}$ This finding has been similarly reported by Touzé et al. ${ }^{30}$ Smoking cessation is crucial in patients harboring these pseudoaneurysms. Hyperlipidemia is also related to vessel wall weakening and thus the promotion of pseudoaneurysm formation and growth. Some studies reported that traumatic dissections have a higher tendency to result in pseudoaneurysm formation and enlargement in comparison with nontraumatic dissections..$^{9,16,18}$ In a retrospective study of 34 aneurysms, Mokri reported that traumatic dissecting aneurysms were common, and significantly fewer traumatic aneurysms resolved or became smaller. ${ }^{16}$ Dissecting pseudoaneurysms of the extracranial ICA did not enlarge in $90 \%$ of patients (30\% remained stable, $40 \%$ decreased in size, and $20 \%$ resolved). ${ }^{16}$ Traumatic dissecting aneurysms of the extracranial ICA did not change in $36 \%$ of patients, decreased in size in $14 \%$, and resolved in $7 \%$. The remaining patients were treated with surgery (29\% of patients with traumatic aneurysms underwent surgical intervention in comparison with $0 \%$ of the spontaneous pseudoaneurysms). ${ }^{16}$ With regard to location, several studies have reported that aneurysms in the vertebral circulation have a better overall anatomical and clinical outcome. ${ }^{15,17,30}$ More specifically, in this study, pseudoan- 
TABLE 4. Significant results in the multivariate analysis

\begin{tabular}{|c|c|c|c|c|c|c|c|c|c|c|c|c|}
\hline \multirow[b]{2}{*}{ Clinical Variable } & \multicolumn{3}{|c|}{ Had an Intervention } & \multicolumn{3}{|c|}{$\begin{array}{l}\text { Pseudoaneurysm } \\
\text { Enlargement }\end{array}$} & \multicolumn{3}{|c|}{ Occurrence of Symptoms } & \multicolumn{3}{|c|}{ Clinical Outcome } \\
\hline & OR & $p$ Value & $95 \% \mathrm{Cl}$ & OR & $p$ Value & $95 \% \mathrm{Cl}$ & OR & $p$ Value & $95 \% \mathrm{Cl}$ & OR & $\mathrm{p}$ Value & $95 \% \mathrm{Cl}$ \\
\hline Initial pseudoaneurysm size & 1.3 & 0.001 & $1.1-1.5$ & 1.4 & 0.001 & $1.16-1.79$ & 1.1 & 0.04 & $1.005-1.2$ & & & \\
\hline $\mathrm{C}_{2}$ location & 5.4 & 0.01 & $1.4-20.1$ & 15 & 0.01 & $1.7-141.7$ & 4.4 & 0.026 & $1.2-16.1$ & & & \\
\hline Patient age & 0.9 & 0.04 & $0.87-0.99$ & & & & & & & & & \\
\hline Hyperlipidemia & 7.2 & 0.02 & $1.4-38.3$ & 17.5 & 0.01 & $1.8-171.9$ & & & & & & \\
\hline Smoking & & & & 15.8 & 0.018 & $1.6-156.6$ & & & & 3.6 & 0.04 & $1.02-13$ \\
\hline Trauma & & & & 26.8 & 0.009 & $2.3-313.3$ & & & & & & \\
\hline $\begin{array}{l}\text { Pseudoaneurysm enlarge- } \\
\text { ment }\end{array}$ & 58 & $<0.001$ & $8-424$ & & & & & & & & & \\
\hline $\begin{array}{l}\text { Any pseudoaneurysm- } \\
\text { related symptoms }\end{array}$ & 32 & $<0.001$ & $9.5-107.9$ & & & & & & & & & \\
\hline
\end{tabular}

eurysm location in the intracranial petrous segment of the ICA had the worst anatomical outcome.

Overall, $3 \%$ of patients had a recurrent TIA, no patients had a recurrent stroke, and $14.2 \%$ of patients had recurrence of nonischemic symptoms (headache, neck pain, Horner syndrome, or cranial nerve palsy). Several potential symptoms resulting from pseudoaneurysm formation have been reported in the literature resulting from compression, TIAs, stroke, or rupture. ${ }^{1,3}$ The low rate of these events in this study is consistent with previous reports that failed to find an association between pseudoaneurysm development and poor outcome or recurrent events in patients with carotid and vertebral dissection..$^{12,30}$ Guillon et al. did not observe any compressive, ischemic, or hemorrhagic symptoms in their study. Only a minority had a poor outcome. ${ }^{12}$ Djouhri et al. did not observe any stroke or TIAs during follow-up. ${ }^{8}$ Benninger et al. observed 3 of 38 stroke events during follow-up. ${ }^{2}$ This reinforces the notion that most pseudoaneurysms have a benign outcome with a low risk of ischemic events and rupture during follow-up. Furthermore, larger aneurysms and those located in the $\mathrm{C}_{2}$ segment of the ICA were significantly associated with the occurrence of recurrent symptoms. It is important to have a close follow-up for these larger aneurysms in the $\mathrm{C}_{2}$ segment as early detection can lead to decreased overall morbidity.

Medical treatment with antiplatelet or anticoagulation therapy was sufficient as a management strategy for most dissecting pseudoaneurysms. Overall, 20.8\% of patients had an intervention other than medical treatment. Some authors have advocated against surgical treatment of dissecting pseudoaneurysms, while others have highly encouraged endovascular and surgical management. ${ }^{11,21,25,30}$ Rao et al. reported 2 cases that did not need any surgical intervention. ${ }^{23}$ Chaer et al. reported a case of a carotid pseudoaneurysm that was initially managed conservatively but was later treated with stenting and coil embolization due to enlargement. ${ }^{6}$ Foreman et al. reported in their study that 2 of $26(7.7 \%)$ traumatic aneurysms were treated with the placement of a covered stent due to evidence of significant aneurysm growth..$^{10}$ Of 12 pseudoaneurysms studied by Pelkonen et al., 8 remained stable and 2 required surgical ligation. ${ }^{20}$ The present study further shows that in patients who were placed on antithrombotic treatment, about one-fifth still underwent an endovascular or surgical intervention during follow-up. Aneurysm enlargement and occurrence of ischemic or nonischemic symptoms were obvious predictors of intervention. Other significant factors that were associated with higher odds of intervention included larger initial size (with pseudoaneurysms $>10 \mathrm{~mm}$ being the most common candidates for intervention), aneurysms located in the $C_{2}$ segment of the ICA, younger patient age, and hyperlipidemia. Larger pseudoaneurysms have a higher risk of resulting in overall symptoms, rupture, and embolization and thus were more likely to be candidates for endovascular or surgical intervention. There was a trend for more aggressive treatment in younger patients in this study, as evidenced by the higher rates of intervention in this population.

Endovascular management with stenting was the most common approach. No complications were seen with endovascular management, and treatment was successful in all pseudoaneurysms with no recurrences observed during follow-up. The only complication that was observed after intervention was seen with the 1 pseudoaneurysm that was surgically clipped. The patient developed hydrocephalus and pseudomeningocele and underwent ventriculoperitoneal shunting. Overall, endovascular interventions were safe for the management of pseudoaneurysms, but were only necessary in about one-fifth of the cases after enlargement or symptom occurrence during follow-up. Similarly, Seward et al. did not observe any complications after endovascular therapy of extracranial carotid artery pseudoaneurysms and reported an overall $8.6 \%$ complication rate after literature review. ${ }^{27}$ Because of the potential complications observed with endovascular and surgical treatment as reported in the literature, pseudoaneurysms that are asymptomatic and non-enlarging would be best managed by medical treatment and close observation without the need for more invasive treatments (Fig. 1).

The main limitations of this study include the fact that it is based on a single center's experience, which may not always reflect the results of other institutions, and the retrospective nature of this study. Since all patients were placed on antithrombotic treatment, the results do not reflect the true natural history of dissecting pseudoaneurysms. But, 


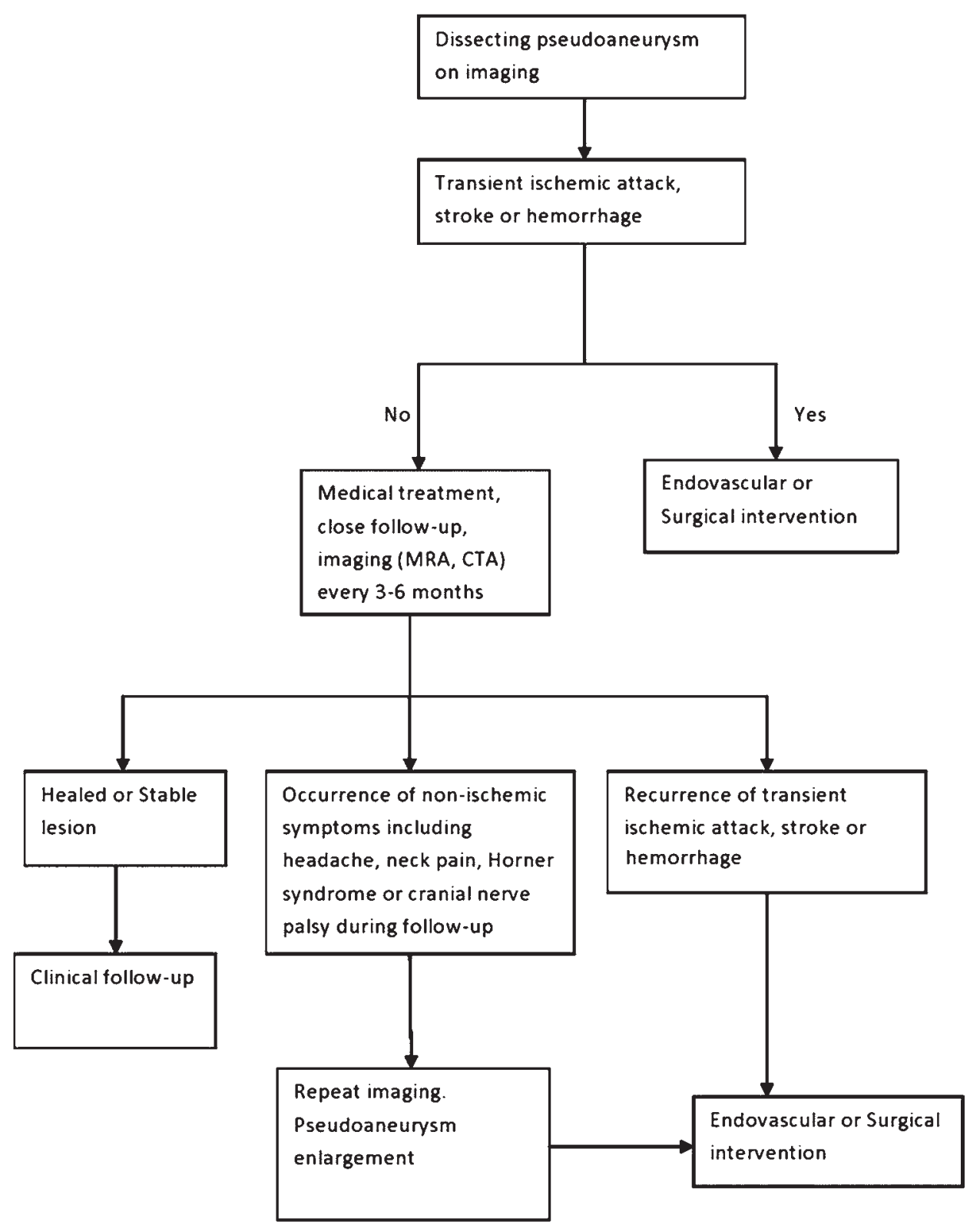

FIG. 1. Follow-up of patients with dissecting pseudoaneurysms. CTA = CT angiography.

since most patients with dissecting pseudoaneurysms are placed on antithrombotic treatment in clinical practice, it does reflect the actual progression of these structures. Clinical outcome determination by the treating neurosurgeon constitutes another limitation of the study.

\section{Conclusions}

Dissecting pseudoaneurysms have a benign course and most will not cause symptoms or enlarge on follow-up. Medical treatment can be sufficient as an initial treatment for dissecting pseudoaneurysms. The minority of pseudoaneurysms that enlarge and cause symptoms can be managed safely with stenting or other endovascular approaches. Aneurysm size and location, history of smoking, and hyperlipidemia are important factors that can influence the progression and treatment of these structures.

\section{References}

1. Baumgartner RW, Arnold M, Baumgartner I, Mosso M, Gönner F, Studer A, et al: Carotid dissection with and without ischemic events: local symptoms and cerebral artery findings. Neurology 57:827-832, 2001

2. Benninger DH, Gandjour J, Georgiadis D, Stöckli E, Arnold $\mathrm{M}$, Baumgartner RW: Benign long-term outcome of conservatively treated cervical aneurysms due to carotid dissection. Neurology 69:486-487, 2007

3. Biousse V, D'Anglejan-Chatillon J, Touboul PJ, Amarenco P, Bousser MG: Time course of symptoms in extracranial 
carotid artery dissections. A series of 80 patients. Stroke 26:235-239, 1995

4. Boström K, Liliequist B: Primary dissecting aneurysm of the extracranial part of the internal carotid and vertebral arteries. A report of three cases. Neurology 17:179-186, 1967

5. Caplan LR, Zarins CK, Hemmati M: Spontaneous dissection of the extracranial vertebral arteries. Stroke 16:1030-1038, 1985

6. Chaer RA, Derubertis B, Kent KC, McKinsey JF: Endovascular treatment of traumatic carotid pseudoaneurysm with stenting and coil embolization. Ann Vasc Surg 22:564-567, 2008

7. Debette S, Grond-Ginsbach C, Bodenant M, Kloss M, Engelter S, Metso T, et al: Differential features of carotid and vertebral artery dissections: the CADISP study. Neurology 77:1174-1181, 2011

8. Djouhri H, Guillon B, Brunereau L, Lévy C, Bousson V, Biousse $\mathrm{V}$, et al: MR angiography for the long-term follow-up of dissecting aneurysms of the extracranial internal carotid artery. AJR Am J Roentgenol 174:1137-1140, 2000

9. Fabian TC, Patton JH Jr, Croce MA, Minard G, Kudsk KA, Pritchard FE: Blunt carotid injury. Importance of early diagnosis and anticoagulant therapy. Ann Surg 223:513-525, 1996

10. Foreman PM, Griessenauer CJ, Falola M, Harrigan MR: Extracranial traumatic aneurysms due to blunt cerebrovascular injury. J Neurosurg 120:1437-1445, 2014

11. Garg K, Rockman CB, Lee V, Maldonado TS, Jacobowitz GR, Adelman MA, et al: Presentation and management of carotid artery aneurysms and pseudoaneurysms. J Vasc Surg 55:1618-1622, 2012

12. Guillon B, Brunereau L, Biousse V, Djouhri H, Lévy C, Bousser MG: Long-term follow-up of aneurysms developed during extracranial internal carotid artery dissection. Neurology 53:117-122, 1999

13. Kadkhodayan Y, Jeck DT, Moran CJ, Derdeyn CP, Cross DT III: Angioplasty and stenting in carotid dissection with or without associated pseudoaneurysm. AJNR Am J Neuroradiol 26:2328-2335, 2005

14. Lévy C, Laissy JP, Raveau V, Amarenco P, Servois V, Bousser MG, et al: Carotid and vertebral artery dissections: threedimensional time-of-flight MR angiography and MR imaging versus conventional angiography. Radiology 190:97-103, 1994

15. Mas JL, Bousser MG, Hasboun D, Laplane D: Extracranial vertebral artery dissections: a review of 13 cases. Stroke 18:1037-1047, 1987

16. Mokri B: Traumatic and spontaneous extracranial internal carotid artery dissections. J Neurol 237:356-361, 1990

17. Mokri B, Houser OW, Sandok BA, Piepgras DG: Spontaneous dissections of the vertebral arteries. Neurology 38:880 885,1988

18. Mokri B, Piepgras DG, Houser OW: Traumatic dissections of the extracranial internal carotid artery. J Neurosurg 68:189197, 1988

19. Nguyen Bui L, Brant-Zawadzki M, Verghese P, Gillan G: Magnetic resonance angiography of cervicocranial dissection. Stroke 24:126-131, 1993

20. Pelkonen O, Tikkakoski T, Leinonen S, Pyhtinen J, Lepojärvi M, Sotaniemi K: Extracranial internal carotid and vertebral artery dissections: angiographic spectrum, course and prognosis. Neuroradiology 45:71-77, 2003

21. Perez-Cruet MJ, Patwardhan RV, Mawad ME, Rose JE: Treatment of dissecting pseudoaneurysm of the cervical internal carotid artery using a wall stent and detachable coils: case report. Neurosurgery 40:622-626, 1997

22. Provenzale JM: Dissection of the internal carotid and vertebral arteries: imaging features. AJR Am J Roentgenol 165:1099-1104, 1995

23. Rao AS, Makaroun MS, Marone LK, Cho JS, Rhee R, Chaer RA: Long-term outcomes of internal carotid artery dissection. J Vasc Surg 54:370-375, 2011

24. Schievink WI: Spontaneous dissection of the carotid and vertebral arteries. N Engl J Med 344:898-906, 2001

25. Schievink WI, Piepgras DG, McCaffrey TV, Mokri B: Surgical treatment of extracranial internal carotid artery dissecting aneurysms. Neurosurgery 35:809-816, 1994

26. Schievink WI, Prakash UB, Piepgras DG, Mokri B: Alpha 1 -antitrypsin deficiency in intracranial aneurysms and cervical artery dissection. Lancet 343:452-453, 1994

27. Seward CJ, Dumont TM, Levy EI: Endovascular therapy of extracranial carotid artery pseudoaneurysms: case series and literature review. J Neurointerv Surg 7:682-689, 2015

28. Song Y, Wang Y, Li C, Wang Y, Mu S, Yang X: Retreatment and outcomes of recurrent intracranial vertebral artery dissecting aneurysms after stent assisted coiling: a single center experience. PLoS One 9:e113027, 2014

29. Stringer WL, Kelly DL Jr: Traumatic dissection of the extracranial internal carotid artery. Neurosurgery 6:123-130, 1980

30. Touzé E, Randoux B, Méary E, Arquizan C, Meder JF, Mas JL: Aneurysmal forms of cervical artery dissection: associated factors and outcome. Stroke 32:418-423, 2001

\section{Disclosures}

The authors report no conflict of interest concerning the materials or methods used in this study or the findings specified in this paper.

\section{Author Contributions}

Conception and design: Tjoumakaris, Daou, Hammer, Chalouhi, Jabbour, Rosenwasser. Acquisition of data: Daou, Hammer, Chalouhi, Starke. Analysis and interpretation of data: Tjoumakaris, Daou, Chalouhi, Starke. Drafting the article: Tjoumakaris, Daou, Hammer, Chalouhi. Critically revising the article: Tjoumakaris, Daou, Hammer, Chalouhi, Jabbour, Rosenwasser. Reviewed submitted version of manuscript: Tjoumakaris, Daou, Jabbour. Approved the final version of the manuscript on behalf of all authors: Tjoumakaris. Statistical analysis: Starke. Study supervision: Tjoumakaris, Jabbour, Rosenwasser.

\section{Correspondence}

Stavropoula I. Tjoumakaris, Division of Neurovascular Surgery and Endovascular Neurosurgery, Department of Neurological Surgery, Thomas Jefferson University Hospital, 909 Walnut St., 2nd Fl., Philadelphia, PA 19107. email: stavropoula. tjoumakaris@jefferson.edu. 